\title{
Tetranitro-oxacalix[4]crown-Based Host-Guest Recognition Motif and A Related [2]Rotaxane-Based Molecular Switch
}

\author{
Hua Liu, ${ }^{\dagger}$ Wen-Jing Hu, ${ }^{\dagger}$ Yahu A. Liu, ${ }^{+}$Jiu-Sheng Li, ${ }^{*}{ }^{\dagger}$ Biao Jiang ${ }^{* \dagger}$ and Ke Wen ${ }^{\prime}$ \\ ${ }^{\dagger}$ Shanghai Advanced Research Institute, Chinese Academy of Science, Shanghai 201210, China \\ ${ }^{*}$ Medicinal Chemistry, ChemBridge Research Laboratories, San Diego, CA 92127, USA
}

* E-Mail: lijs@sari.ac.cn, jiangb@sari.ac.cn, wenk@sari.ac.cn 
Table of Contents

1. NMR spectra (including 2D 1H NMR Of R1) of new compounds S3-S8

2. Figure S1. ${ }^{1} \mathrm{H}$ NMR spectra of mixed solution of $\mathrm{H1}$ and $\mathrm{G1}$ in acetone- $d_{6} \quad$ S9

3. Figure S2. ${ }^{1} \mathrm{H}$ NMR spectra of mixed solution of $\mathrm{H2}$ and $\mathrm{G} 2$ in $\mathrm{CDCl}_{3} \quad \mathrm{S9}$

4. Figure S3. 2D NOESY spectrum of G2СH1 S10

5. Figure S4. Computer model of the threaded complex G2 $\subset$ H1 generated by Gaussian $09 \quad$ S11

6. Table S1. The Cartesian coordinates of the optimized geometry of G2 $\subset \mathrm{H1} \quad$ S11-S15

7. Figure S5-S8. Job plot based on ${ }^{1} \mathrm{H}$ NMR data, ${ }^{1} \mathrm{H}$ NMR titration and association constant $\begin{array}{ll}\text { determination of the complex G2ᄃH1 } & \text { S15-S17 }\end{array}$

5. Figure S9. ${ }^{1} \mathrm{H}$ NMR spectra of R1, H1 and T1 S18

6 References $\quad$ S18 


\section{NMR spectra of related compounds}

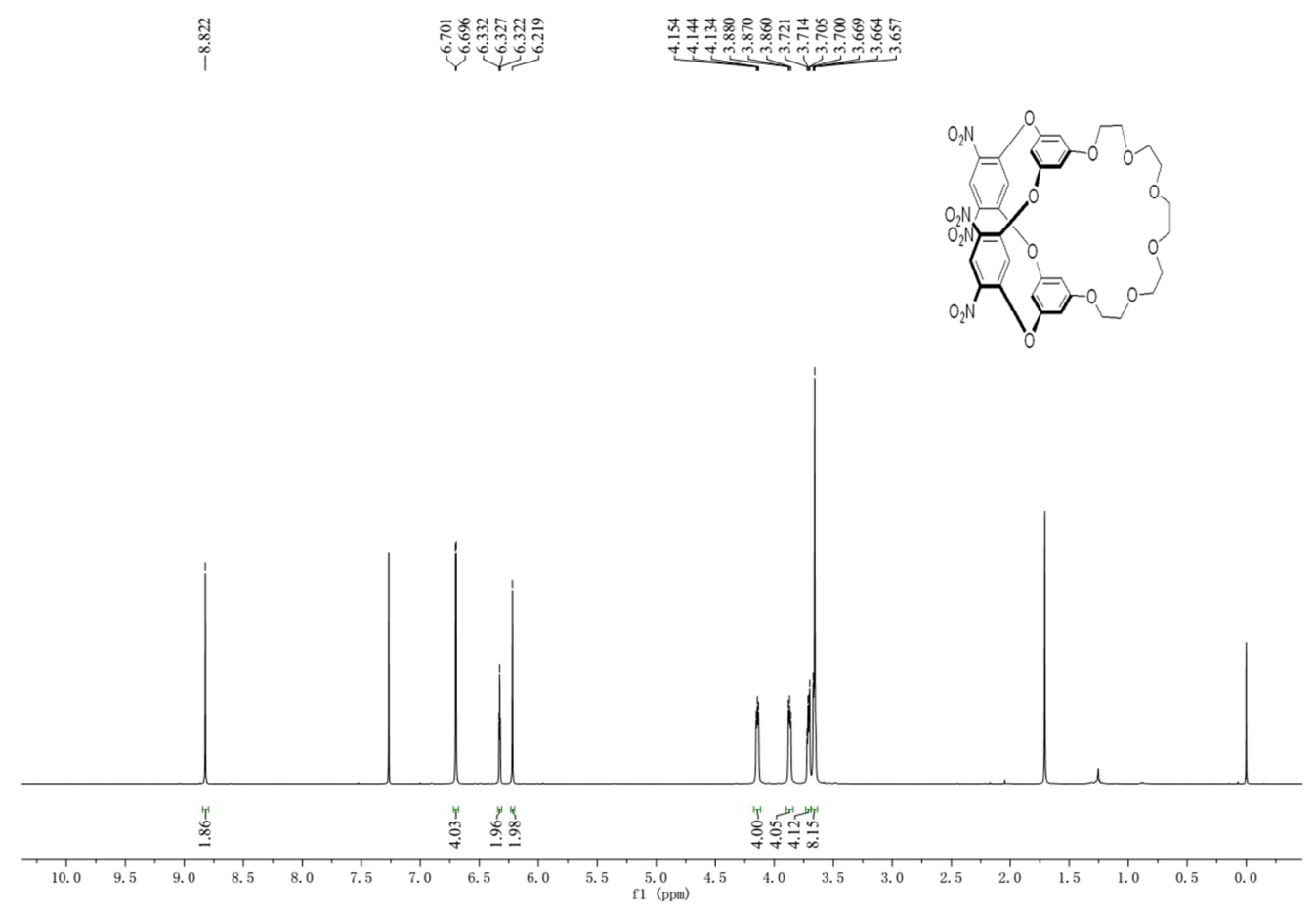

${ }^{1} \mathrm{H}$ NMR spectrum of compound $\mathbf{H 1}$
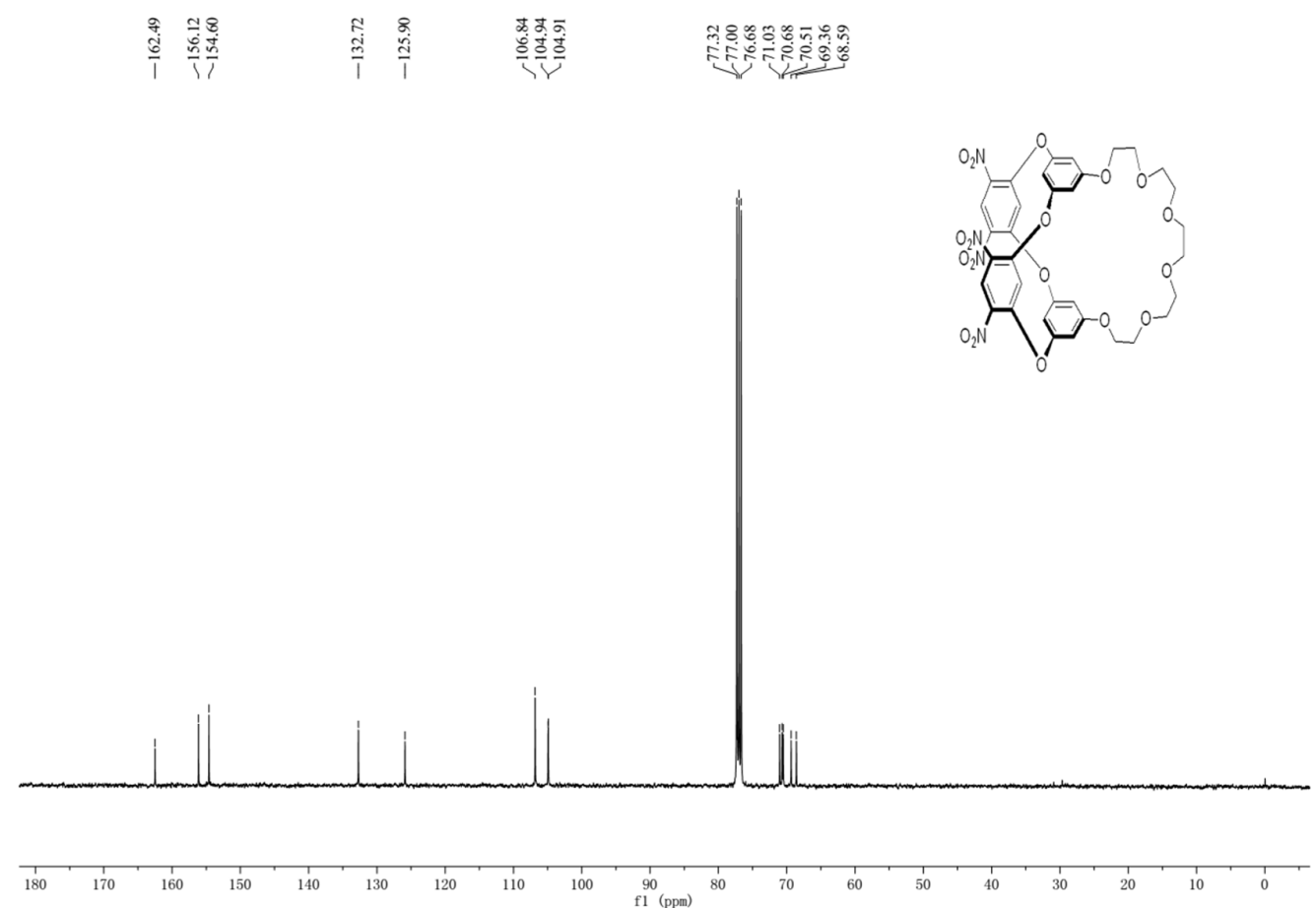

${ }^{13} \mathrm{C}$ NMR spectrum of compound $\mathbf{H 1}$ 


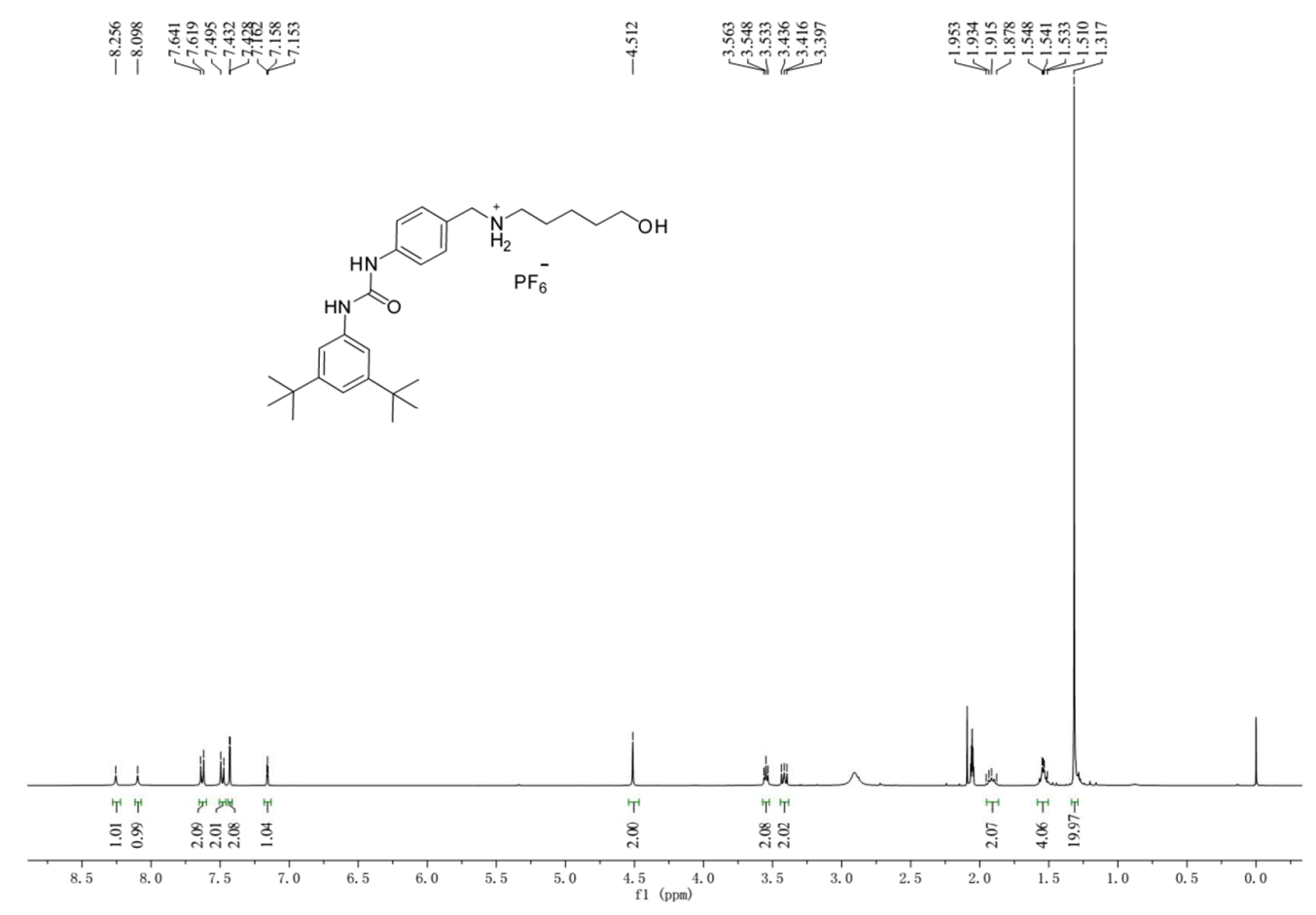

${ }^{1}$ H NMR spectrum of compound $\mathbf{T}$

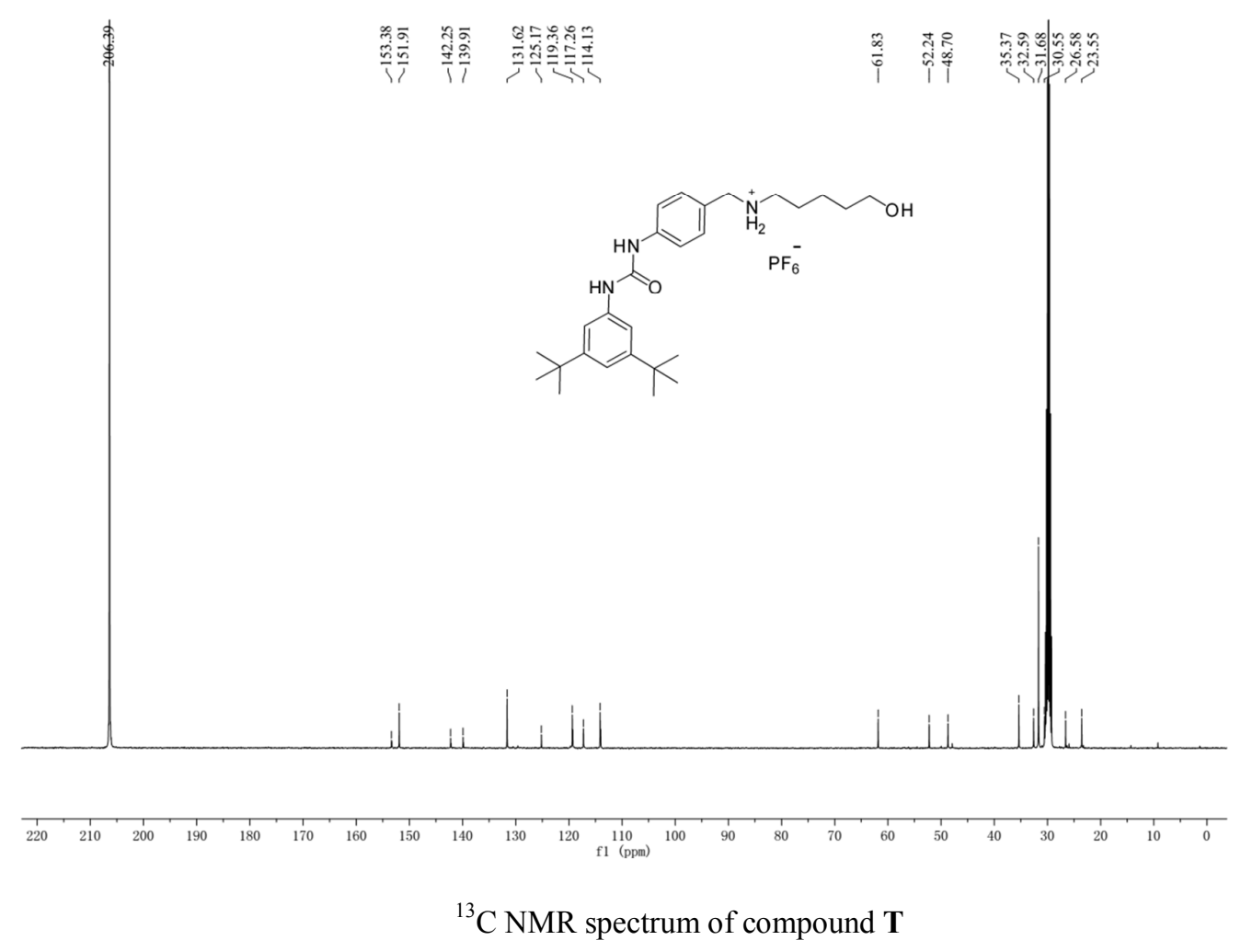




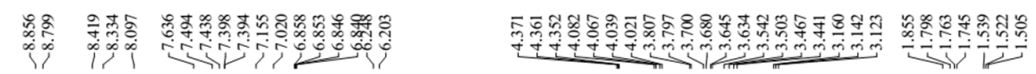
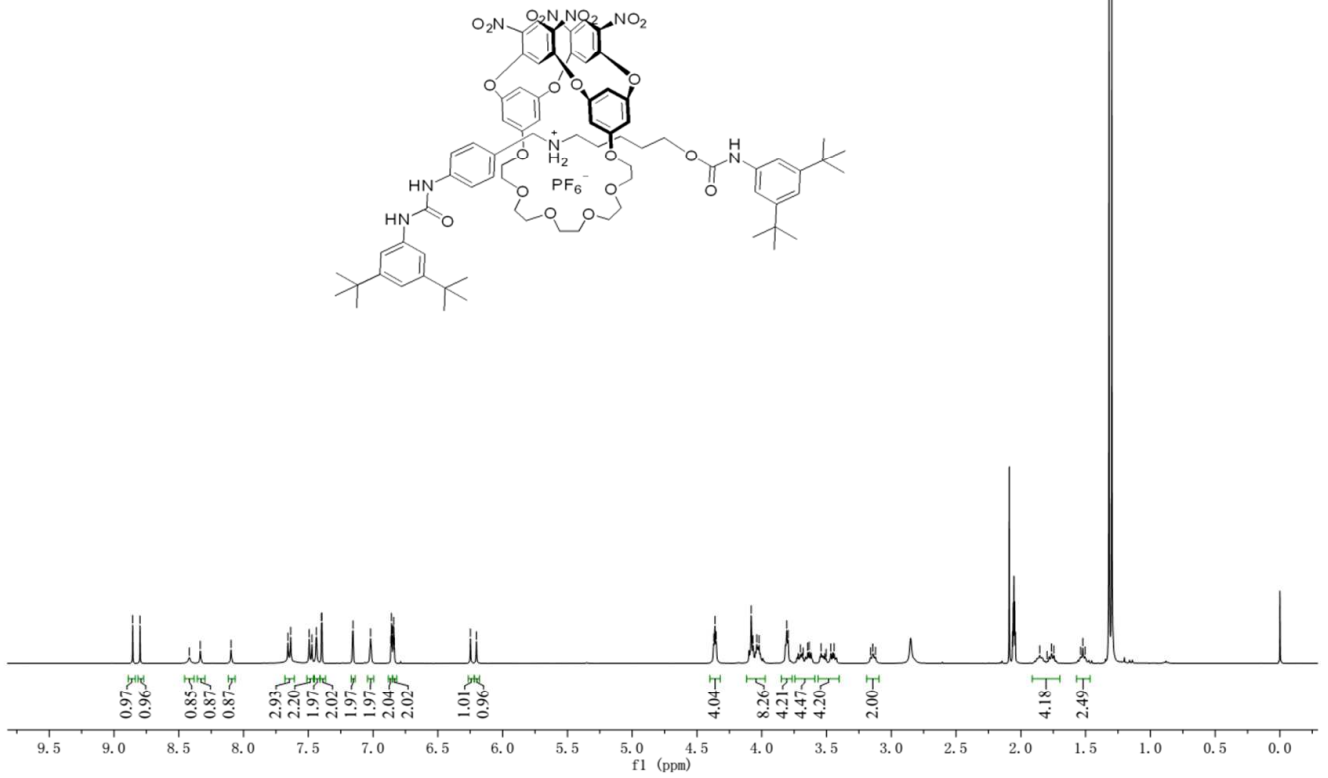

${ }^{1}$ H NMR spectrum of compound R1
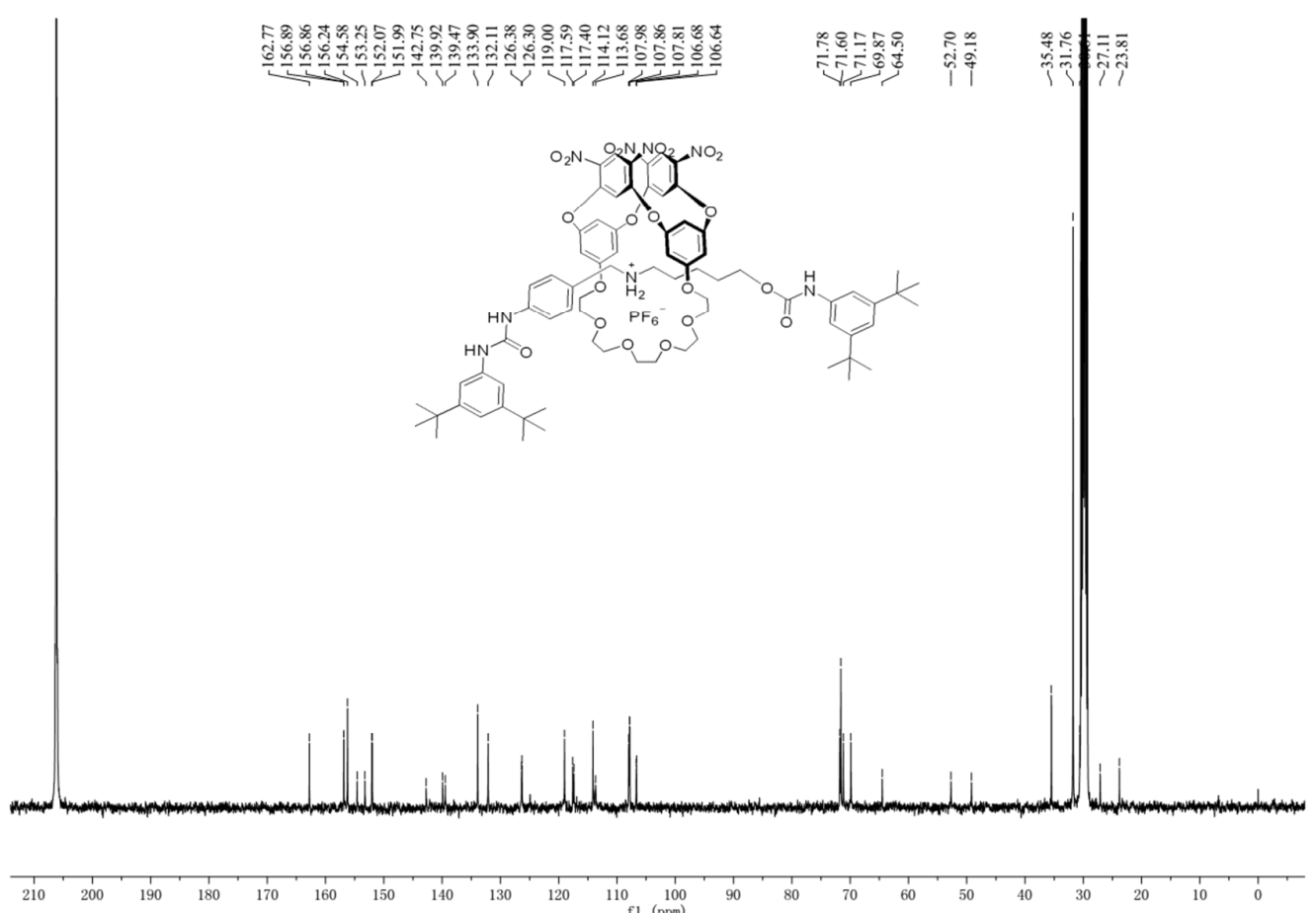

${ }^{13} \mathrm{C}$ NMR spectrum of compound $\mathbf{R} \mathbf{1}$ 


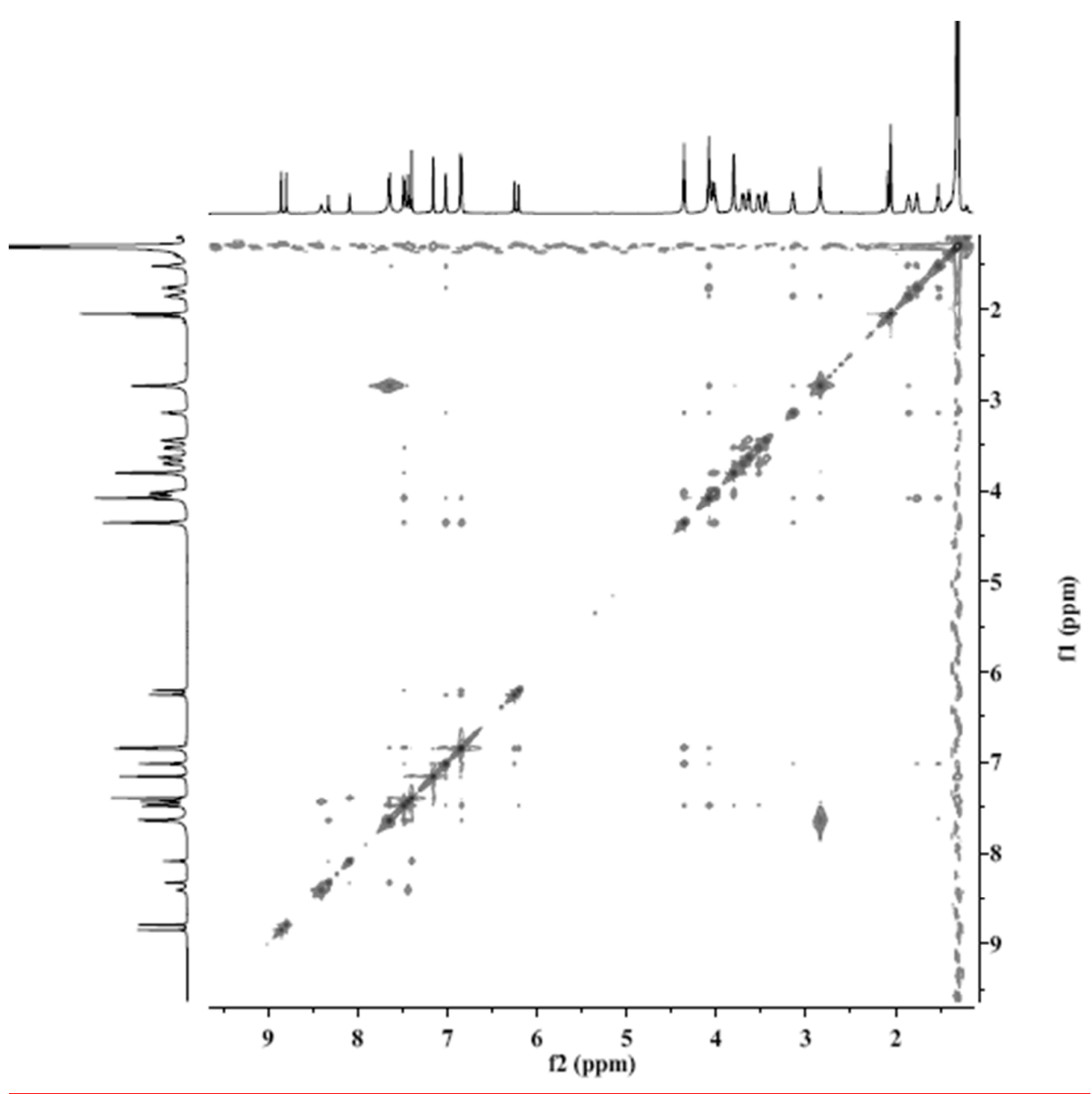

Partial 2D NOESY spectrum of rotaxane R1 (10 mM) in acetone- $d_{6}(400 \mathrm{MHz}, 298 \mathrm{~K})$ 


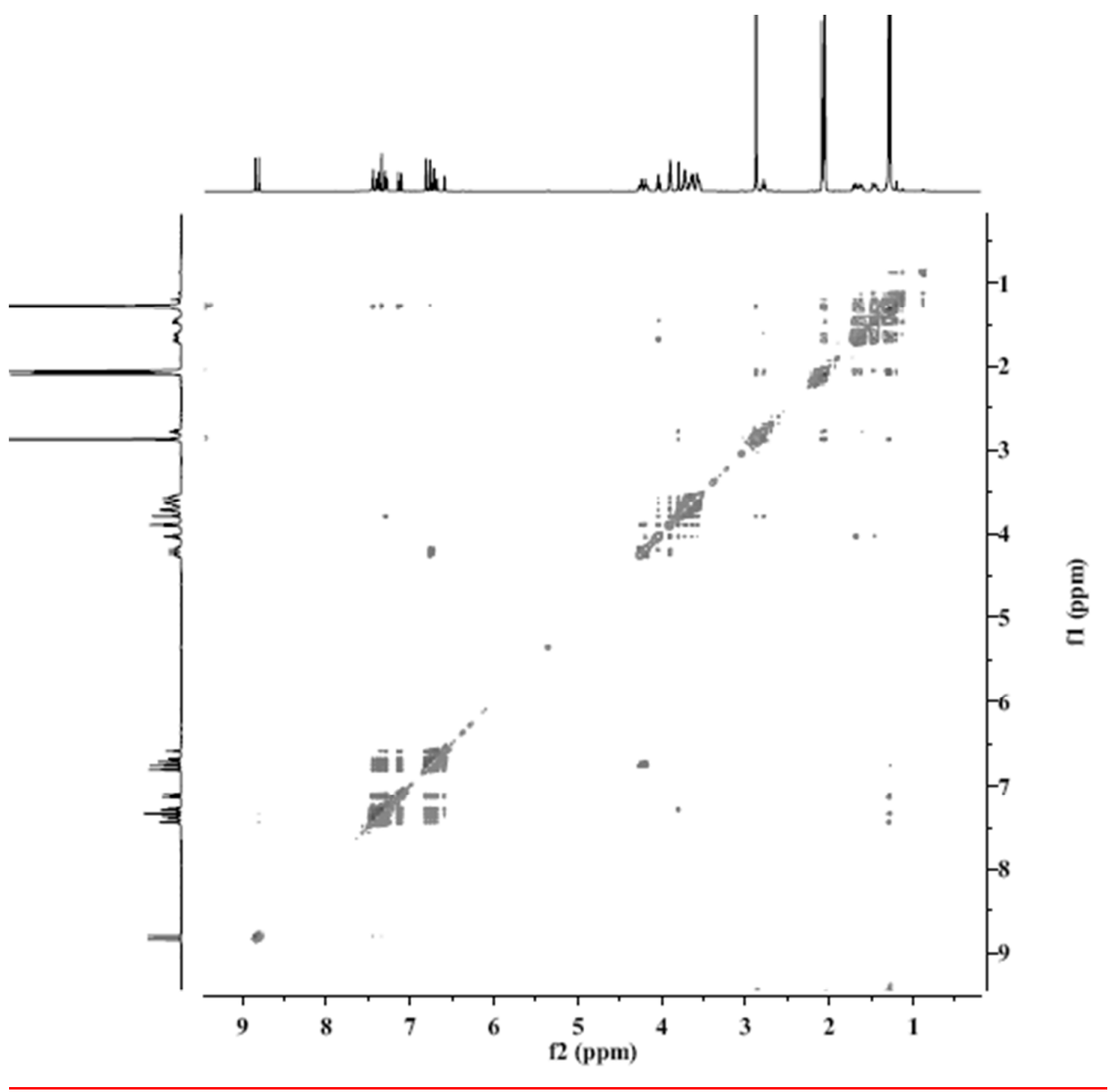

Partial 2D NOESY spectrum of the mixture of rotaxane R1 $(10 \mathrm{mM})$ and DABCO $(20 \mathrm{mM})$ in acetone- $d_{6}$ (400 MHz, $298 \mathrm{~K})$ 


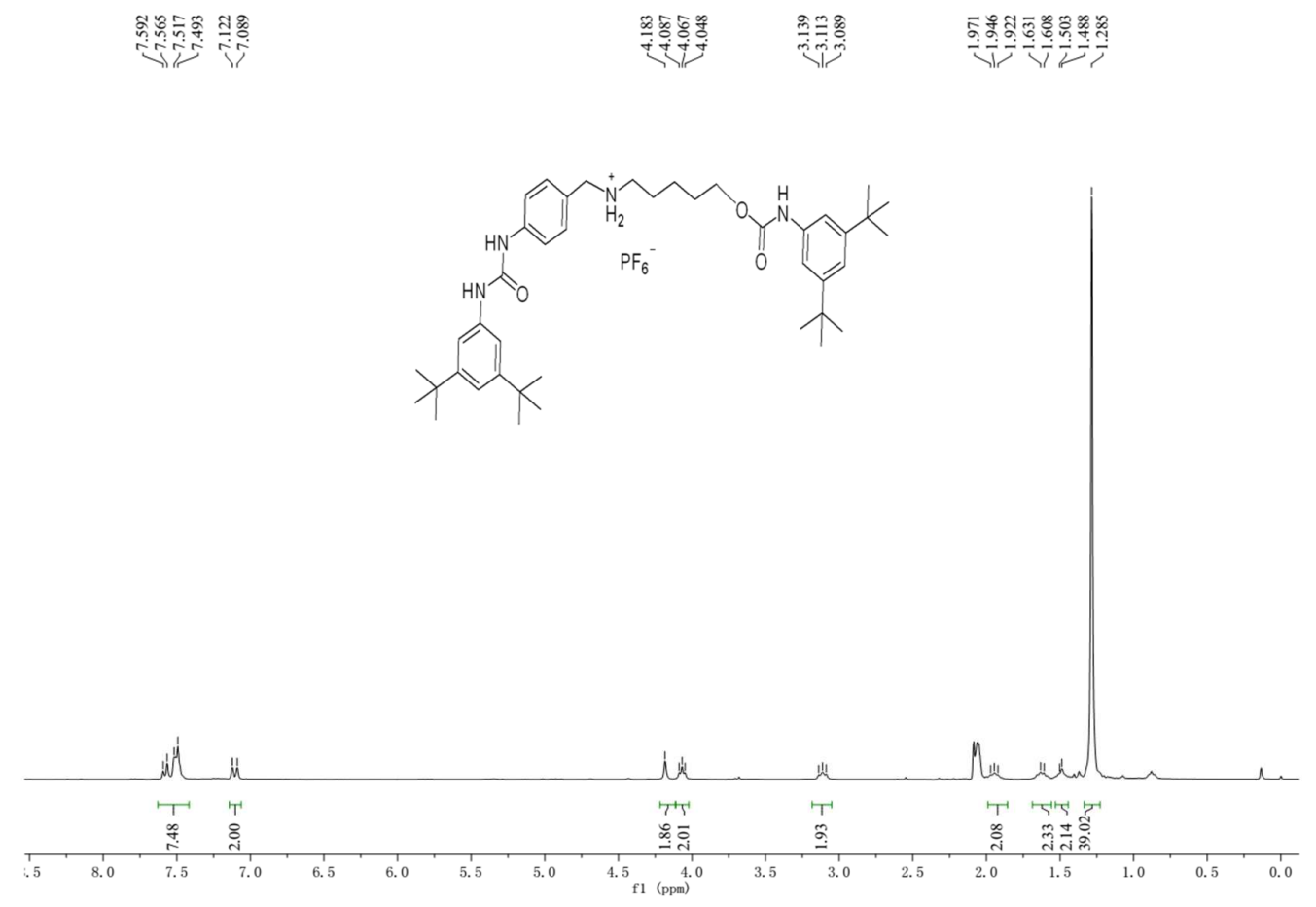

${ }^{1}$ H NMR spectrum of compound T1
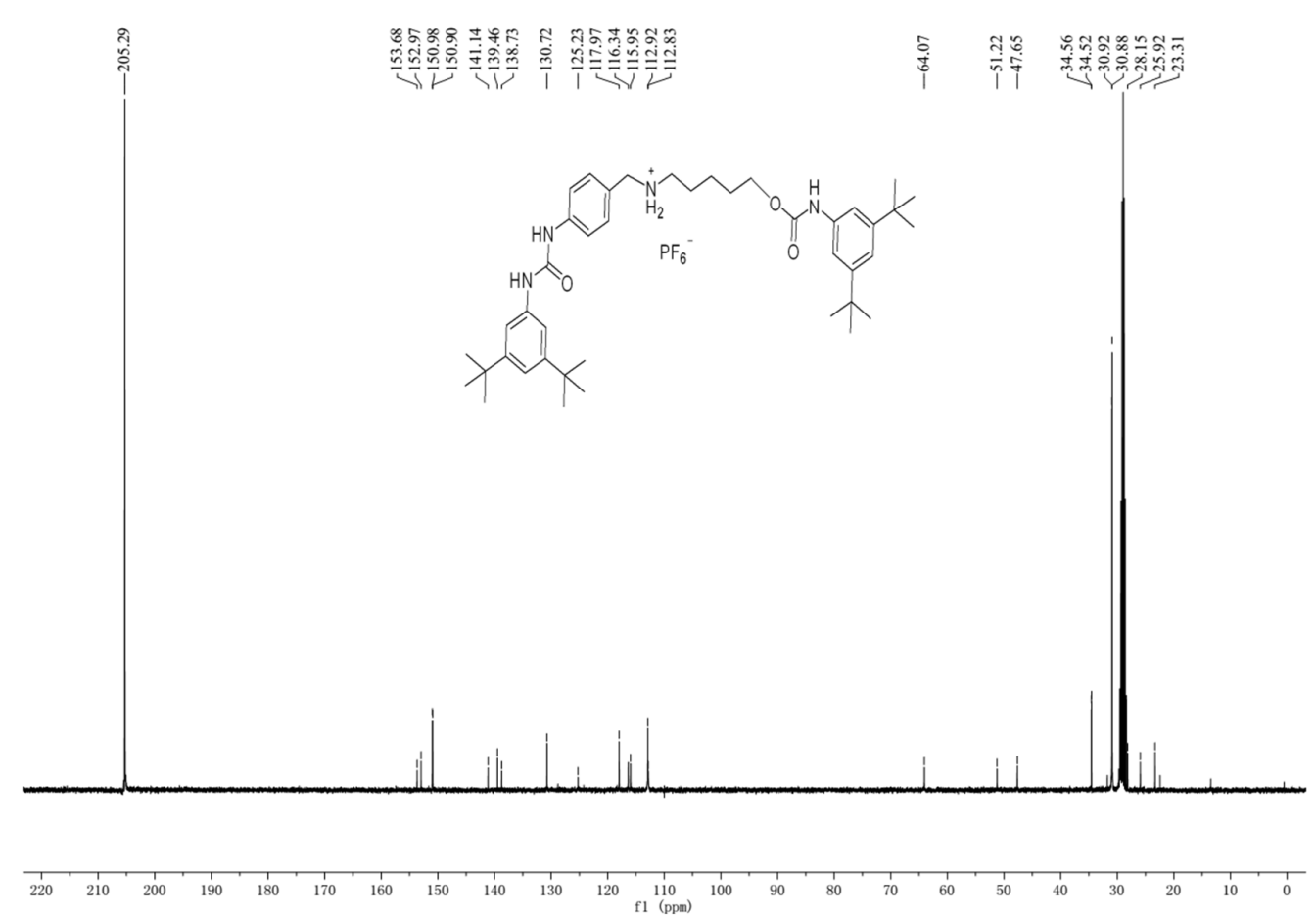

${ }^{13} \mathrm{C}$ NMR spectrum of compound $\mathbf{T} \mathbf{1}$ 


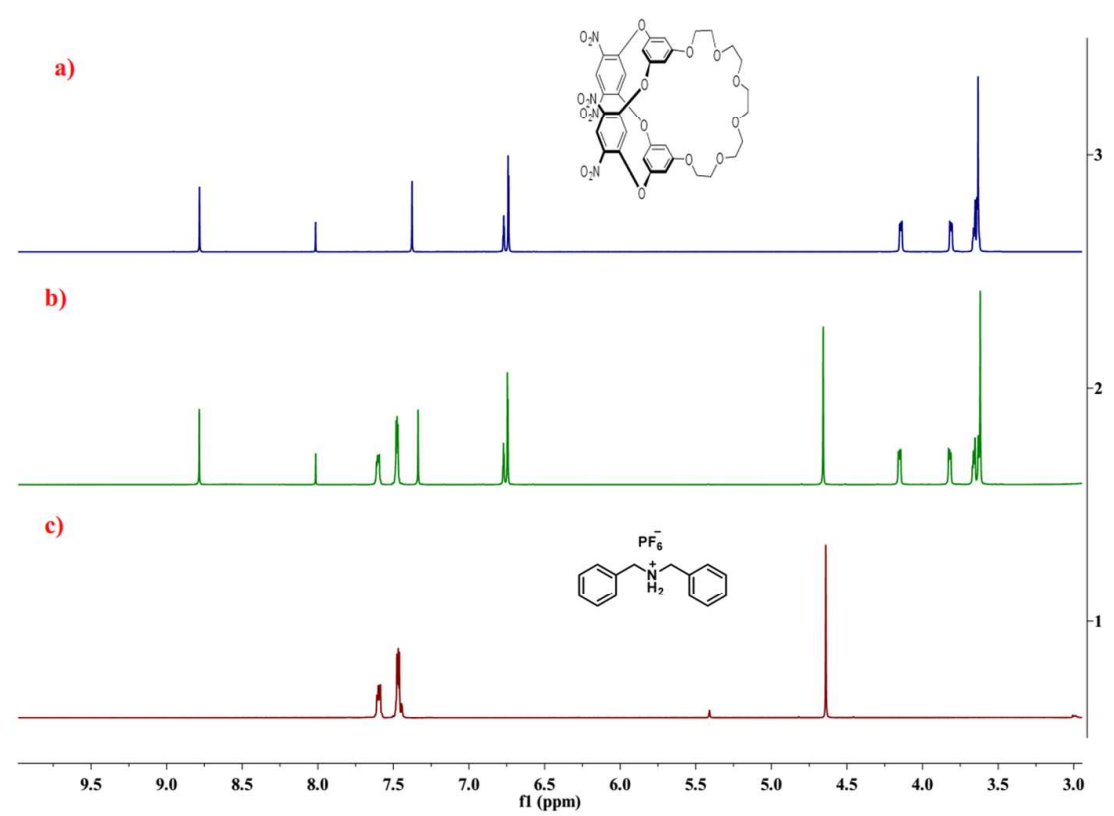

Figure S1. ${ }^{1} \mathrm{H}$ NMR spectra (500 MHz, acetone- $d_{6}, 298 \mathrm{~K}$ ): (a) H1, (b) H1 + G1, (c) G1, $c=10 \mathrm{mM}$. Spectrum b shows no change in chemical shifts after mix of $\mathbf{H 1}$ and G1, suggesting no complexion occurred between $\mathbf{H 1}$ and G1.

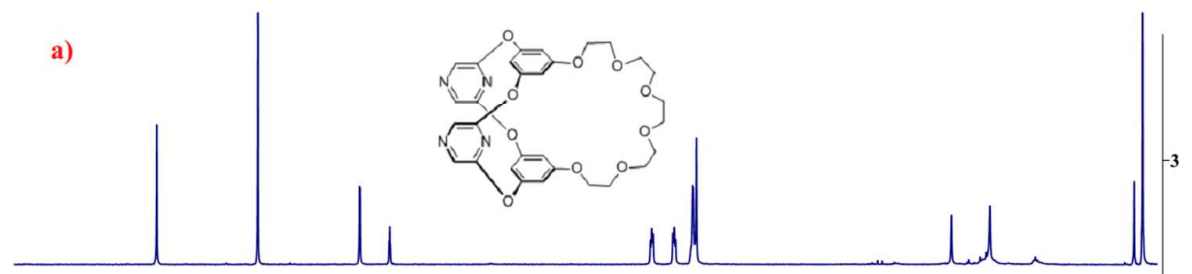

b)

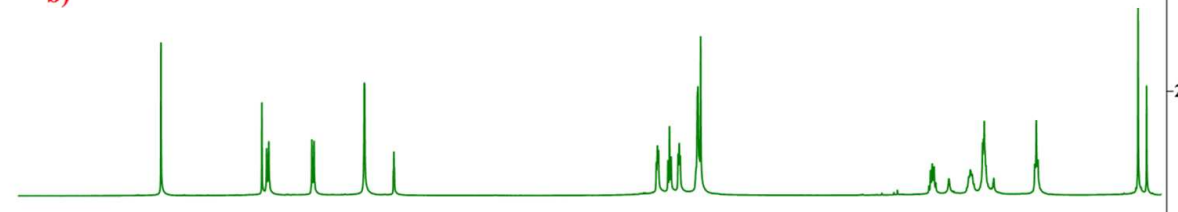

c)
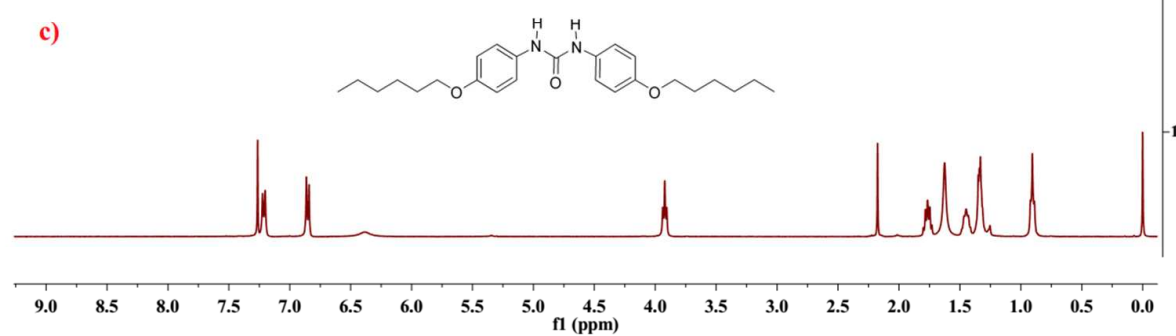

Figure S2. ${ }^{1} \mathrm{H}$ NMR spectra $\left(500 \mathrm{MHz}, \mathrm{CDCl}_{3}, 298 \mathrm{~K}\right.$ ): (a) $\mathbf{H 2}$, (b) $\mathbf{H 2}+\mathbf{G 2}$, (c) $\mathbf{G 2}, c=10 \mathrm{mM}$. Spectrum $b$ showed no change in chemical shifts after mixing of $\mathbf{H 2}$ and $\mathbf{G} \mathbf{2}$, suggesting no complexion occurred between $\mathbf{H} \mathbf{2}$ and $\mathbf{G 2}$. 


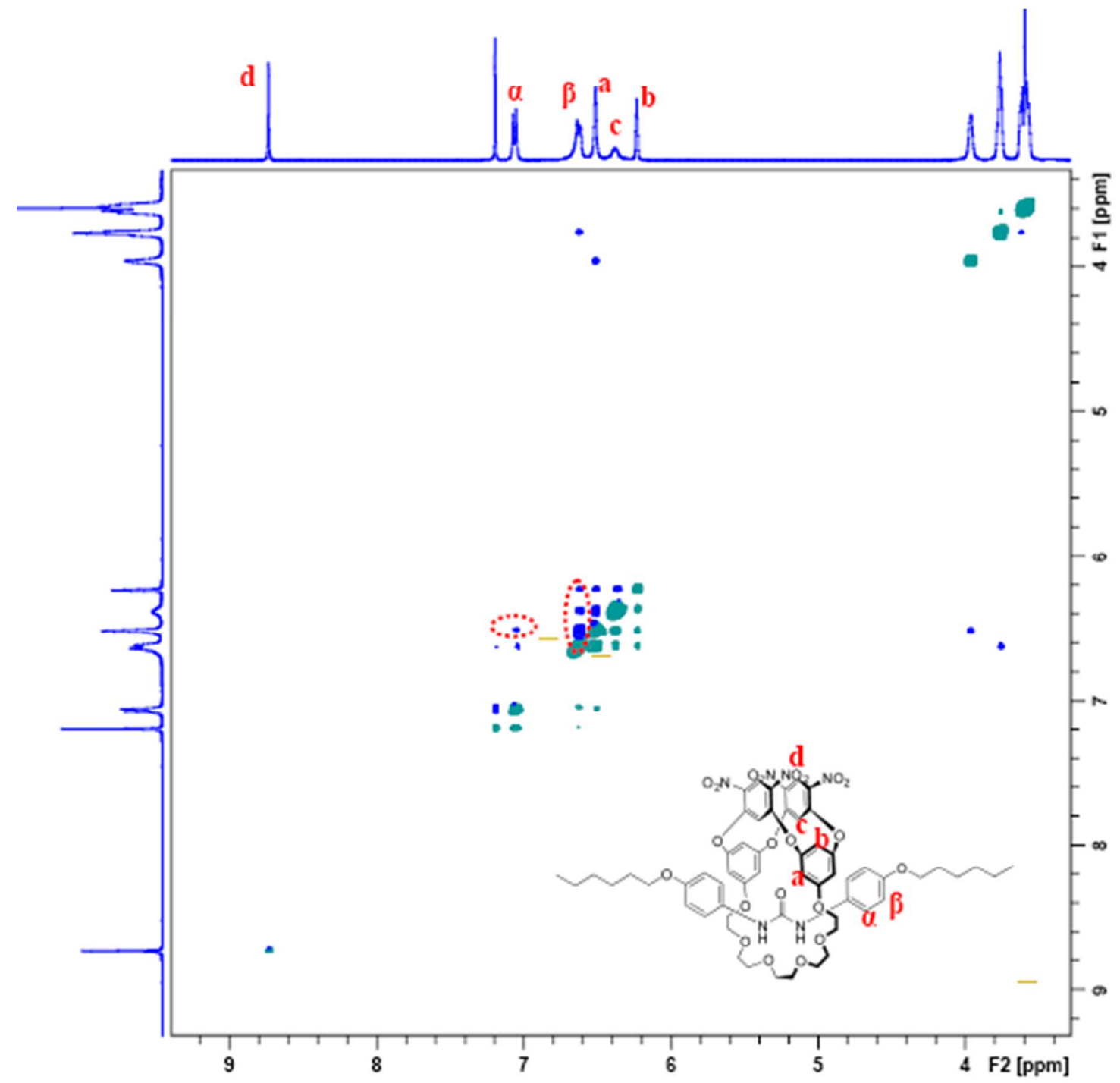

Figure S3. Partial 2D NOESY spectrum of a mixture of $\mathbf{H 1}(10 \mathrm{mM})$ and $\mathbf{G 2}(10 \mathrm{mM})$ in $\mathrm{CDCl}_{3}(400$ MHz, $298 \mathrm{~K}$ ), Correlations of proton signals between H1 and G2 were clearly observed, suggesting the complexion between $\mathbf{H 1}$ and $\mathbf{G 2}$. 


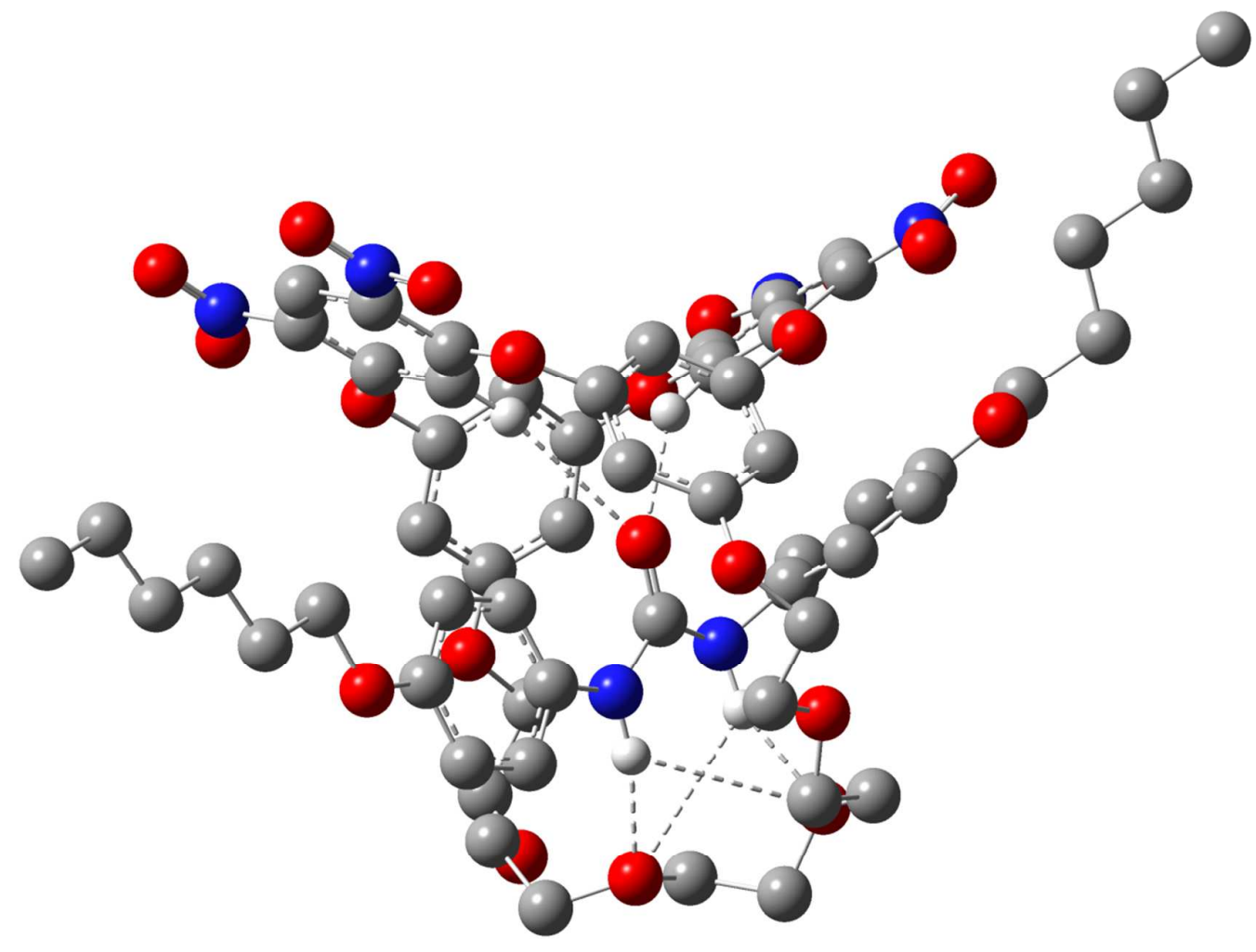

Figure S4. Computer model of the threaded complex G2CH1. The structure of the molecule was initially drawn using the AGUI graphical interface from the AMPAC program package. ${ }^{1}$ The equilibrium geometry and vibrational frequencies of the molecule were calculated using the semi-empirical PM6 method ${ }^{2}$ as implemented in the Gaussian 09 program package. ${ }^{3}$ The calculated vibrational frequencies showed that the optimized structure is indeed a minimum. The Cartesian coordinates of the optimized geometry was given in Table S1. The optimized structure was rendered using the Materials Visualizer graphical interface from the Materials Studio program package. ${ }^{4}$

Table S1. Cartesian coordinates in Angstroms for the optimized structure of the complex G2сH1.

$\begin{array}{llll}\mathrm{C} & -2.856060 & -2.993851 & 0.252172 \\ \mathrm{C} & -2.229720 & -2.159303 & 1.181974 \\ \mathrm{C} & -2.627737 & -2.217550 & 2.520828 \\ \mathrm{C} & -3.651531 & -3.120199 & 2.945959 \\ \mathrm{C} & -4.265253 & -3.947817 & 1.999959 \\ \mathrm{C} & -3.895292 & -3.890252 & 0.651125 \\ \mathrm{H} & -1.456158 & -1.448820 & 0.860909 \\ \mathrm{H} & -5.057578 & -4.653900 & 2.321189 \\ \mathrm{C} & 2.990934 & -1.155041 & 1.664295 \\ \mathrm{C} & 2.219760 & -1.368452 & 0.522945\end{array}$




\begin{tabular}{|c|c|c|c|}
\hline $\mathrm{C}$ & 2.785240 & -2.040975 & -0.561438 \\
\hline $\mathrm{C}$ & 4.121837 & -2.545853 & -0.501405 \\
\hline $\mathrm{C}$ & 4.887450 & -2.298952 & 0.642704 \\
\hline $\mathrm{C}$ & 4.348311 & -1.598314 & 1.729850 \\
\hline $\mathrm{H}$ & 1.212834 & -0.921493 & 0.440535 \\
\hline $\mathrm{H}$ & 5.936097 & -2.654147 & 0.685212 \\
\hline $\mathrm{C}$ & -0.777434 & -0.714065 & -3.263265 \\
\hline $\mathrm{C}$ & 0.563965 & -0.963603 & -2.930332 \\
\hline $\mathrm{C}$ & 0.806645 & -1.897836 & -1.924516 \\
\hline $\mathrm{C}$ & -0.191720 & -2.575277 & -1.222248 \\
\hline $\mathrm{C}$ & -1.503526 & -2.278174 & -1.608275 \\
\hline $\mathrm{C}$ & -1.840394 & -1.369454 & -2.602166 \\
\hline $\mathrm{H}$ & 1.390380 & -0.473230 & -3.442859 \\
\hline $\mathrm{H}$ & 0.036101 & -3.314178 & -0.459821 \\
\hline $\mathrm{H}$ & -2.876197 & -1.168218 & -2.877246 \\
\hline $\mathrm{C}$ & -1.346187 & 0.794350 & 3.506244 \\
\hline $\mathrm{C}$ & -0.263886 & 1.702208 & 3.442771 \\
\hline $\mathrm{C}$ & 1.039743 & 1.274213 & 3.147036 \\
\hline $\mathrm{C}$ & 1.225656 & -0.089371 & 2.912420 \\
\hline $\mathrm{C}$ & 0.205305 & -1.040474 & 2.954882 \\
\hline $\mathrm{C}$ & -1.064814 & -0.543688 & 3.268928 \\
\hline $\mathrm{H}$ & -2.350465 & 1.138121 & 3.752937 \\
\hline $\mathrm{H}$ & 1.887266 & 1.952336 & 3.083622 \\
\hline $\mathrm{H}$ & 0.390812 & -2.099128 & 2.798271 \\
\hline $\mathrm{N}$ & -4.584484 & -4.750973 & -0.317207 \\
\hline $\mathrm{O}$ & -4.314140 & -4.613985 & -1.493862 \\
\hline $\mathrm{O}$ & -5.399192 & -5.539951 & 0.134101 \\
\hline $\mathrm{N}$ & -4.078836 & -3.195780 & 4.348999 \\
\hline $\mathrm{N}$ & 5.200303 & -1.288515 & 2.876532 \\
\hline $\mathrm{N}$ & 4.722948 & -3.273385 & -1.623433 \\
\hline $\mathrm{O}$ & -3.544661 & -2.452843 & 5.147650 \\
\hline $\mathrm{O}$ & -4.953511 & -4.006032 & 4.610137 \\
\hline $\mathrm{O}$ & 4.679184 & -0.886483 & 3.896872 \\
\hline $\mathrm{O}$ & 6.406107 & -1.437755 & 2.716562 \\
\hline $\mathrm{O}$ & 4.089798 & -3.355470 & -2.656850 \\
\hline $\mathrm{O}$ & 5.832354 & -3.751760 & -1.435703 \\
\hline $\mathrm{O}$ & -2.596142 & -3.011748 & -1.078120 \\
\hline $\mathrm{O}$ & 2.167416 & -2.252205 & -1.751521 \\
\hline $\mathrm{O}$ & 2.577008 & -0.483682 & 2.772217 \\
\hline $\mathrm{O}$ & -2.141986 & -1.433636 & 3.513016 \\
\hline $\mathrm{O}$ & -1.195424 & 0.114459 & -4.265176 \\
\hline $\mathrm{O}$ & -0.634611 & 2.991293 & 3.687838 \\
\hline $\mathrm{C}$ & 1.931590 & 4.762936 & -2.799488 \\
\hline $\mathrm{C}$ & 0.512757 & 4.398856 & -3.238044 \\
\hline
\end{tabular}




\begin{tabular}{|c|c|c|c|}
\hline $\mathrm{C}$ & -0.304141 & 1.160216 & -4.748581 \\
\hline $\mathrm{C}$ & -0.674514 & 2.439113 & -3.999303 \\
\hline $\mathrm{H}$ & -1.284837 & 2.221446 & -3.098619 \\
\hline $\mathrm{H}$ & 2.389052 & 5.537046 & -3.436691 \\
\hline $\mathrm{H}$ & 0.187734 & 4.954462 & -4.134497 \\
\hline $\mathrm{C}$ & 1.312206 & 6.440938 & -1.124320 \\
\hline $\mathrm{C}$ & 0.512542 & 6.203260 & 0.152380 \\
\hline $\mathrm{H}$ & 2.131166 & 7.168318 & -0.966089 \\
\hline $\mathrm{H}$ & 0.447295 & 7.108343 & 0.783722 \\
\hline $\mathrm{C}$ & -1.863127 & 6.157982 & 0.644192 \\
\hline $\mathrm{C}$ & -1.792976 & 5.345487 & 1.934591 \\
\hline $\mathrm{H}$ & -1.812207 & 7.244202 & 0.848552 \\
\hline $\mathrm{H}$ & -1.527630 & 4.284779 & 1.766492 \\
\hline $\mathrm{C}$ & 0.392439 & 4.036893 & 3.646679 \\
\hline $\mathrm{C}$ & -0.410027 & 5.307859 & 3.926530 \\
\hline $\mathrm{H}$ & 0.197660 & 6.069013 & 4.455050 \\
\hline $\mathrm{O}$ & 0.595497 & 2.989331 & -3.567161 \\
\hline $\mathrm{O}$ & 1.975186 & 5.187061 & -1.424588 \\
\hline $\mathrm{O}$ & -0.816548 & 5.798225 & -0.284383 \\
\hline $\mathrm{O}$ & -0.746656 & 5.991641 & 2.710093 \\
\hline $\mathrm{H}$ & -1.209166 & 3.159430 & -4.640509 \\
\hline $\mathrm{H}$ & -0.220913 & 4.545112 & -2.421056 \\
\hline $\mathrm{H}$ & 2.589528 & 3.863685 & -2.776474 \\
\hline $\mathrm{H}$ & 0.667501 & 6.778435 & -1.952775 \\
\hline $\mathrm{H}$ & 0.923488 & 5.376209 & 0.762641 \\
\hline $\mathrm{H}$ & -2.776304 & 5.922281 & 0.062607 \\
\hline $\mathrm{H}$ & -2.738032 & 5.398826 & 2.504929 \\
\hline $\mathrm{H}$ & -1.323544 & 5.083090 & 4.511973 \\
\hline $\mathrm{H}$ & 1.140209 & 3.821911 & 4.428785 \\
\hline $\mathrm{H}$ & 0.858634 & 4.041009 & 2.644148 \\
\hline $\mathrm{H}$ & -0.562563 & 1.189628 & -5.824028 \\
\hline $\mathrm{H}$ & 0.762893 & 0.898009 & -4.624600 \\
\hline $\mathrm{C}$ & 0.216497 & 1.993763 & -0.153745 \\
\hline $\mathrm{O}$ & 0.136764 & 0.776032 & -0.035607 \\
\hline $\mathrm{N}$ & 1.425694 & 2.720868 & 0.028870 \\
\hline $\mathrm{H}$ & 1.455867 & 3.706564 & -0.320717 \\
\hline $\mathrm{N}$ & -0.904550 & 2.821174 & -0.379348 \\
\hline $\mathrm{H}$ & -0.780486 & 3.851997 & -0.441010 \\
\hline $\mathrm{C}$ & -2.241259 & 2.345624 & -0.579969 \\
\hline $\mathrm{C}$ & -3.172766 & 3.264702 & -1.120712 \\
\hline $\mathrm{C}$ & -2.639814 & 1.038004 & -0.268427 \\
\hline $\mathrm{C}$ & -4.482902 & 2.871887 & -1.345768 \\
\hline $\mathrm{H}$ & -2.862616 & 4.280819 & -1.364682 \\
\hline $\mathrm{C}$ & -3.956986 & 0.631858 & -0.497756 \\
\hline
\end{tabular}




\begin{tabular}{|c|c|c|c|}
\hline $\mathrm{H}$ & -1.916918 & 0.327330 & 0.147913 \\
\hline $\mathrm{C}$ & -4.868707 & 1.547991 & -1.035039 \\
\hline $\mathrm{H}$ & -5.221844 & 3.555265 & -1.760600 \\
\hline $\mathrm{H}$ & -4.250841 & -0.384661 & -0.254653 \\
\hline $\mathrm{C}$ & 2.690191 & 2.042995 & -0.191801 \\
\hline $\mathrm{C}$ & 2.969031 & 1.395032 & -1.413938 \\
\hline $\mathrm{C}$ & 3.652706 & 2.108387 & 0.822846 \\
\hline $\mathrm{C}$ & 4.209027 & 0.809200 & -1.612859 \\
\hline $\mathrm{H}$ & 2.204239 & 1.359797 & -2.194428 \\
\hline $\mathrm{C}$ & 4.910550 & 1.529092 & 0.630494 \\
\hline $\mathrm{H}$ & 3.427908 & 2.613346 & 1.762220 \\
\hline C & 5.179253 & 0.883435 & -0.584020 \\
\hline $\mathrm{H}$ & 4.461186 & 0.300057 & -2.542265 \\
\hline $\mathrm{H}$ & 5.657494 & 1.593114 & 1.416347 \\
\hline $\mathrm{C}$ & -6.700733 & -0.054289 & -1.086023 \\
\hline $\mathrm{H}$ & -6.113741 & -0.760744 & -1.696490 \\
\hline $\mathrm{H}$ & -6.603748 & -0.286996 & -0.013003 \\
\hline $\mathrm{C}$ & -8.153697 & 0.056733 & -1.540949 \\
\hline $\mathrm{H}$ & -8.188197 & 0.417510 & -2.589471 \\
\hline $\mathrm{H}$ & -8.672133 & 0.837568 & -0.948311 \\
\hline $\mathrm{C}$ & -8.871315 & -1.290755 & -1.408691 \\
\hline $\mathrm{H}$ & -8.348308 & -2.061298 & -2.009515 \\
\hline $\mathrm{H}$ & -8.829583 & -1.643691 & -0.359546 \\
\hline $\mathrm{C}$ & -10.333622 & -1.185980 & -1.861958 \\
\hline $\mathrm{H}$ & -10.862534 & -0.419414 & -1.263363 \\
\hline $\mathrm{H}$ & -10.381085 & -0.833756 & -2.910595 \\
\hline $\mathrm{C}$ & -11.056531 & -2.534386 & -1.734538 \\
\hline $\mathrm{H}$ & -10.530698 & -3.302925 & -2.335049 \\
\hline $\mathrm{H}$ & -11.012198 & -2.889427 & -0.686073 \\
\hline $\mathrm{C}$ & -12.512459 & -2.437564 & -2.183981 \\
\hline $\mathrm{H}$ & -13.019699 & -3.404724 & -2.089810 \\
\hline $\mathrm{H}$ & -13.074489 & -1.713627 & -1.583192 \\
\hline $\mathrm{H}$ & -12.592712 & -2.127248 & -3.231989 \\
\hline $\mathrm{C}$ & 7.462143 & 0.282039 & 0.047853 \\
\hline $\mathrm{H}$ & 7.719366 & 1.334752 & 0.247311 \\
\hline $\mathrm{H}$ & 7.138174 & -0.224565 & 0.975946 \\
\hline $\mathrm{C}$ & 8.567086 & -0.462403 & -0.694163 \\
\hline $\mathrm{H}$ & 9.553529 & -0.131231 & -0.314661 \\
\hline $\mathrm{H}$ & 8.538352 & -0.175137 & -1.768157 \\
\hline $\mathrm{C}$ & 8.428212 & -1.983142 & -0.563101 \\
\hline $\mathrm{H}$ & 8.484637 & -2.281230 & 0.501901 \\
\hline $\mathrm{H}$ & 7.421357 & -2.292368 & -0.920083 \\
\hline $\mathrm{C}$ & 9.499450 & -2.727047 & -1.370103 \\
\hline $\mathrm{H}$ & 10.504669 & -2.514274 & -0.961475 \\
\hline
\end{tabular}




$\begin{array}{lrrr}\mathrm{H} & 9.511328 & -2.359911 & -2.414637 \\ \mathrm{C} & 9.235331 & -4.239948 & -1.360995 \\ \mathrm{H} & 9.246313 & -4.619271 & -0.321080 \\ \mathrm{H} & 8.211061 & -4.441461 & -1.742895 \\ \mathrm{C} & 10.260205 & -4.998975 & -2.200011 \\ \mathrm{H} & 11.279818 & -4.845858 & -1.830324 \\ \mathrm{H} & 10.061602 & -6.076613 & -2.183164 \\ \mathrm{H} & 10.237504 & -4.679902 & -3.248222 \\ \mathrm{O} & -6.189120 & 1.299022 & -1.315683 \\ \mathrm{O} & 6.348332 & 0.256430 & -0.911796\end{array}$

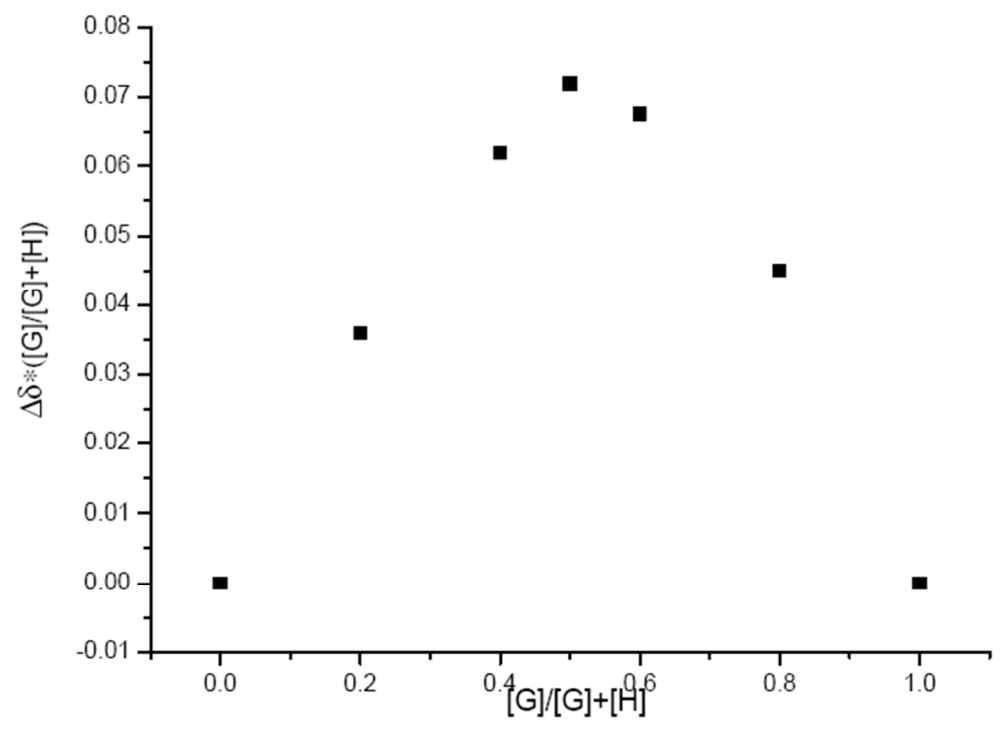

Figure S5. Job plot showing the 1:1 stoichiometry of the complex between $\mathbf{H 1}$ and $\mathbf{G} 2$ in $\mathrm{CDCl}_{3}$ by plotting the $\Delta \delta$ in chemical shift of H1's phenyl proton $\left(\mathrm{H}_{\mathrm{a}}\right)$ signals observed by ${ }^{1} \mathrm{H}$ NMR spectroscopy against the mole fraction of complex. ([host $]+$ [guest $]=20 \mathrm{mM}$ ). 


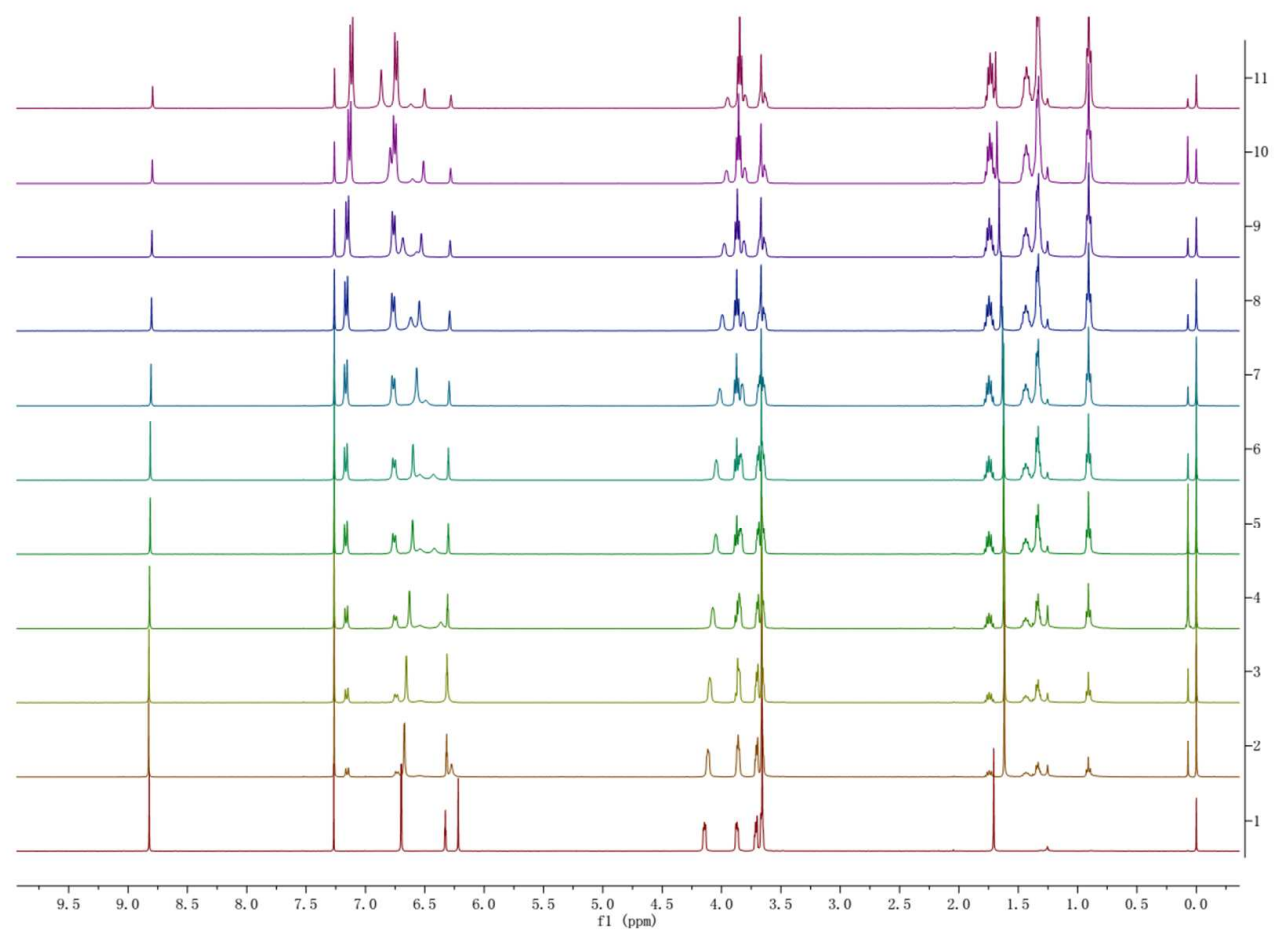

Figure S6. Partial ${ }^{1} \mathrm{H}$ NMR spectra $\left(\mathrm{CDCl}_{3}, 400 \mathrm{MHz}, 298 \mathrm{~K}\right)$ of $\mathbf{H 1}$ at a concentration of $10 \mathrm{mM}$ upon addition of G2. From bottom to top, the concentrations of $\mathbf{G 2}$ were 0, 2, 4, 6, 8, 10, 15, 20, 30, 40, $50 \mathrm{mM}$, respectively.

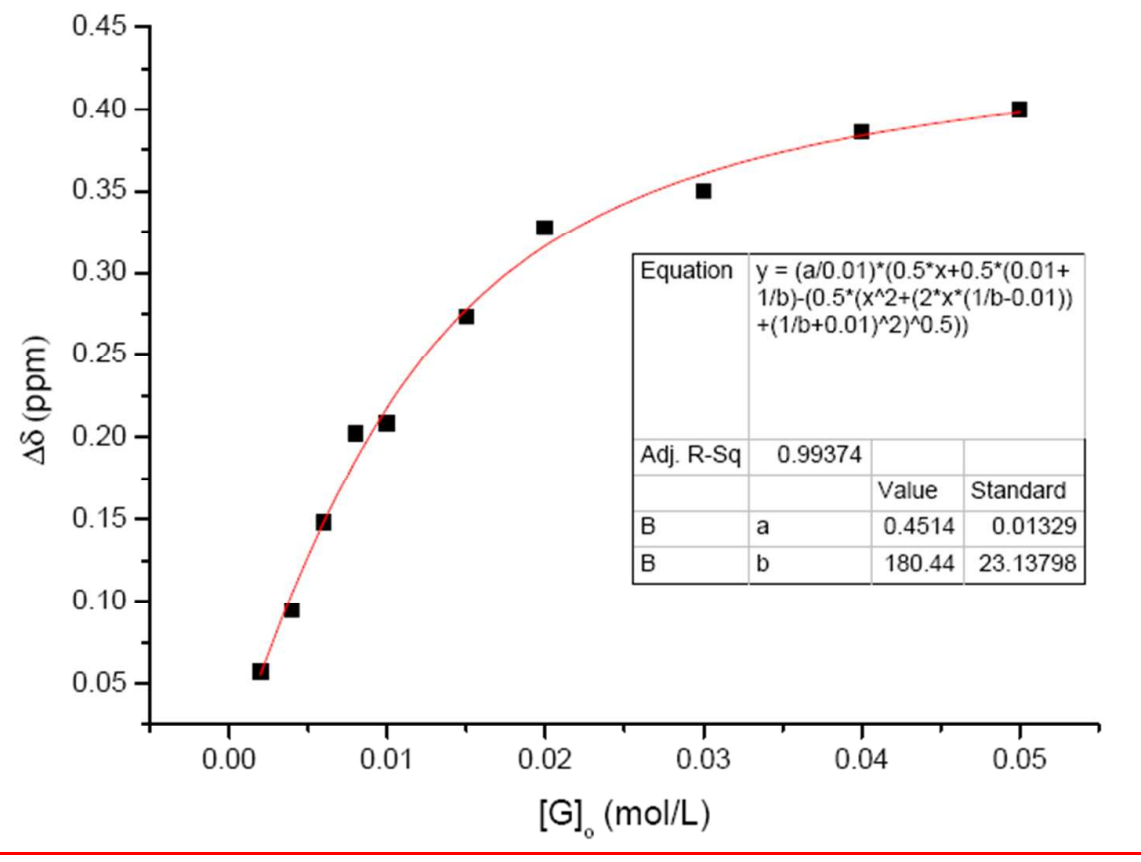

Figure S7. The non-linear curve-fitting (NMR titrations, $\Delta \delta$ of $\mathrm{H}_{\mathrm{c}}$ ) for the complexation of $\mathbf{H 1}(10 \mathrm{mM})$ with $\mathbf{G 2}$ with a 1:1 model. The concentration of $\mathbf{G 2}$ was $0,2,4,6,8,10,15,20,30,40,50 \mathrm{mM}$. The $K_{a}$ value for complex $\mathbf{G} \mathbf{2} \subset \mathbf{H 1}$ in $\mathrm{CDCl}_{3}$ at $298 \mathrm{~K}$ is determined to be $\mathbf{1 8 0} \pm \mathbf{2 3} \mathbf{M}^{\mathbf{- 1}}$. 


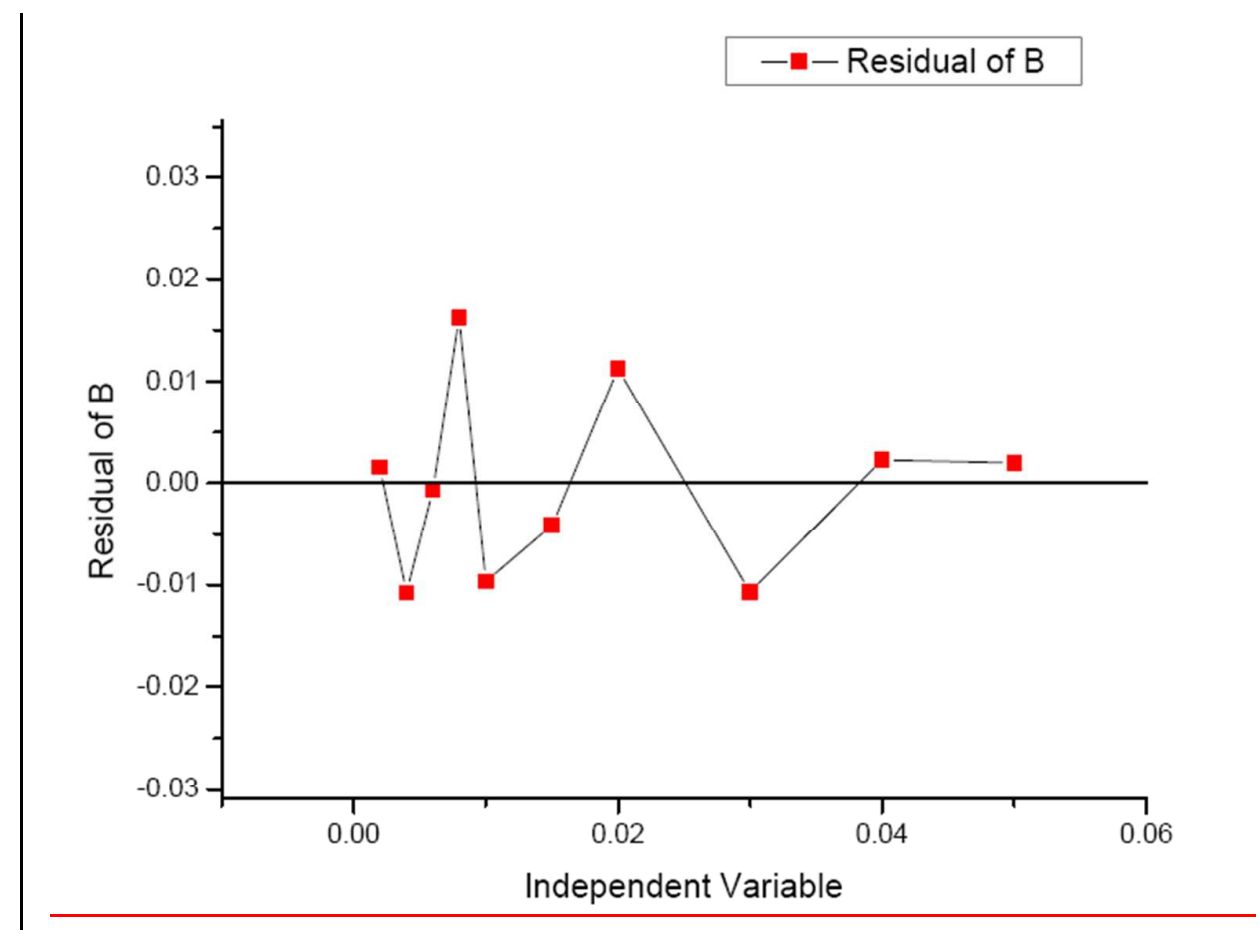

Figure S8. The residual distribution of the corresponding titration data fitted with 1:1 model, which showed a random distribution in a relative small residual range, suggesting the data were fitted with a proper model. ${ }^{6}$ 


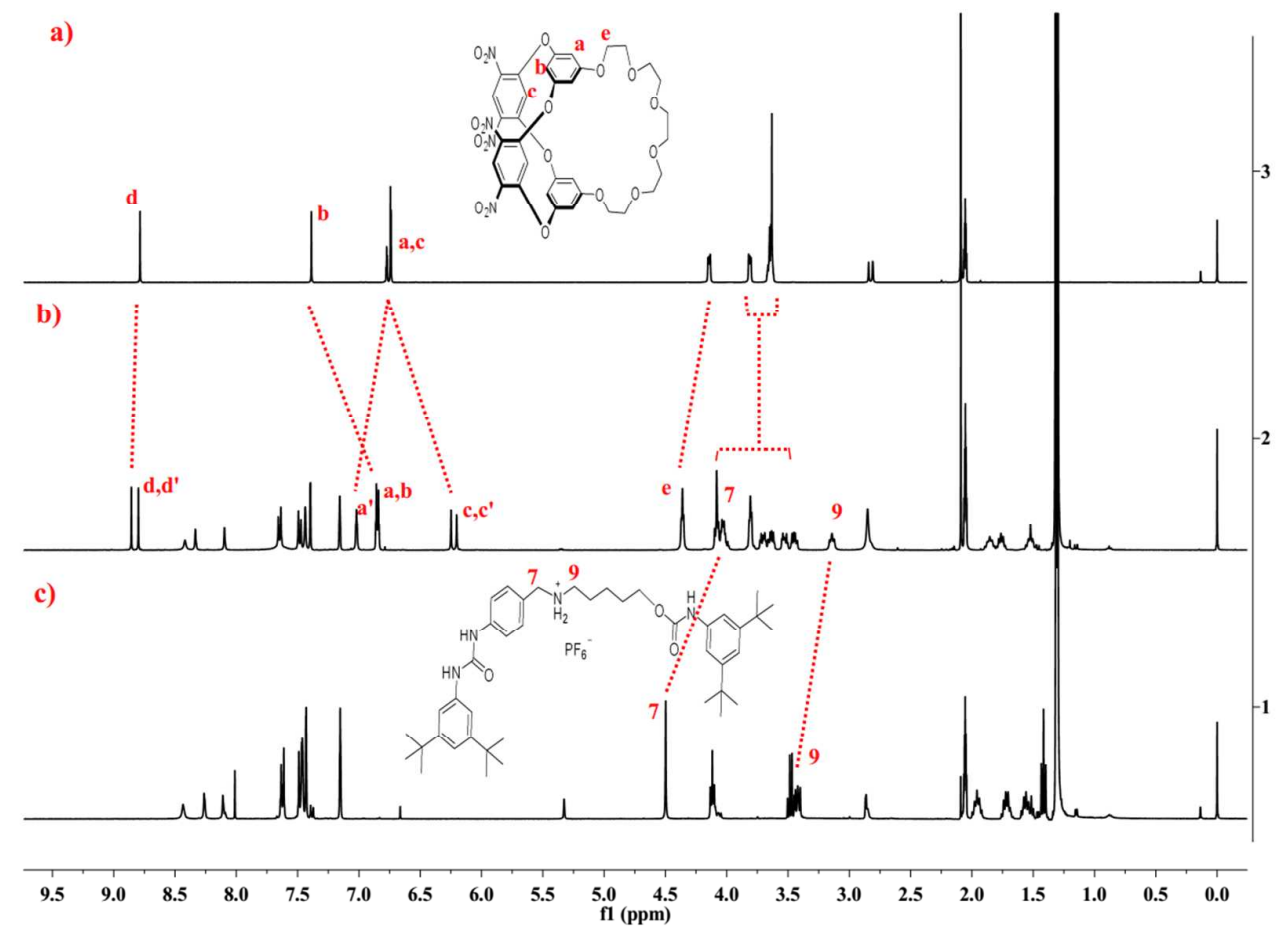

Figure S9. ${ }^{1} \mathrm{H}$ NMR spectra (400 MHz, acetone- $d_{6}, 298 \mathrm{~K}$ ): (a) H1, (b) R1, (c) T1. Some proton signals of $\mathbf{H 1}$ and $\mathbf{T} 1$ components are splitted in rotaxane $\mathbf{R} 1$.

\section{References}

(1) AMPACTM 9, Semichem Inc., Shawnee, KS, 1992-2008.

(2) Stewart, J. J. P. "Optimization of parameters for semiempirical methods. V. Modification of NDDO approximations and application to 70 elements," J. Mol. Model. 2007, 13, 173-213.

(3) Frisch, M. J. et al., Gaussian 09 (revision C.01), Gaussian Inc., Wallingford, CT, 2009.

(4) Materials Studio, Release 6.0, Accelrys Software Inc.: San Diego, 2011.

(5) Shu, X.; Chen, S.; Li, J.; Chen, Z.; Weng, L.; Jia, X.; Li, C. Chem. Commun. 2012, 2967.

(6) Ulatowski, F.; Dąbrowa, K.; Bałakier, T. Jurczak, J. J. Org. Chem. 2016, 81, 1746. 